\title{
Synthesis of $\beta$-Amino Carbonyl Compounds via the Iodine-Alumina Catalyzed Three-Component Coupling Reaction under Microwave Irradiation
}

\author{
Mantu Rajbangshi, Md. Rumum Rohman, Icydora Kharkongor, \\ Hormi Mecadon, and Bekington Myrboh
}

Department of Chemistry, North Eastern Hill University, Mawlai Campus, Shillong 793022, India

Correspondence should be addressed to Bekington Myrboh, bmyrboh@nehu.ac.in

Received 3 September 2010; Accepted 13 December 2010

Academic Editor: Bill Baker

Copyright (c) 2011 Mantu Rajbangshi et al. This is an open access article distributed under the Creative Commons Attribution License, which permits unrestricted use, distribution, and reproduction in any medium, provided the original work is properly cited.

\begin{abstract}
Iodine-alumina was employed as a catalyst in the coupling reactions of aldehydes, enolizable ketones, or 1,3-dicarbonyls with methyl carbamate or aromatic amines under microwave irradiation to afford $\beta$-amino carbonyl compounds in good-to-excellent yields. The key features of this environmentally friendly methodology are its operational simplicity, mild reaction conditions, and less reaction time.
\end{abstract}

\section{Introduction}

In recent years, the use of multicomponent reactions has gained considerable attention in organic synthesis. In particular, the Mannich reaction has been widely used for the synthesis of $\beta$-amino carbonyl compounds. Owing to their importance as valuable building blocks for the preparation of 1,3 -amino alcohols [1,2], $\beta$-amino acids [3], as well as for the synthesis of various bioactive molecules such as the antibiotic nikkomycins and neopolyoxines [4, 5], several methods have been reported in the literature for the synthesis of $\beta$-amino carbonyl compounds using catalysts such as $\mathrm{HClO}_{4}-\mathrm{SiO}_{2}[6]$, silica supported sulfuric acid [7], bromodimethylsulfonium bromide (BDMS) [8], TMSCl [9], p-TSA [10], $\mathrm{Sml}_{3}[11]$, Amberlyst-15 [12], and $\mathrm{AuCl}_{3}-\mathrm{PPh}_{3}$ [13]. These methods however, have certain drawbacks such as moisture sensitivity of the catalyst $[9,10]$, longer reaction time $[6-8]$, and the use of expensive metal salt as catalyst [11-13]. There is still scope, therefore, for an improved method for the synthesis of $\beta$-amino carbonyl compounds which can avoid the use of expensive and sensitive catalysts.

Furthermore, the use of inorganic solid supported reagents provides an attractive procedure due to their characteristic properties such as enhanced reactivity and selectivity, simple workup procedure, and milder reaction conditions [14-17]. Among these inorganic supported reagents, iodine supported on dehydrated neutral alumina has found wide application because of its property to form activated iodonium ion [16]. Therefore, we decided to use activated iodonium ion produced from iodine adsorbed on neutral alumina for the coupling reactions of aldehydes, enolizable ketones or 1,3-dicarbonyls with methyl carbamate or aromatic amines using microwave as an energy source, which is superior to conventional methods [18$20]$ in terms of shorter reaction time and minimization of reaction byproducts. Recently, a method using molecular iodine as the catalyst has been reported for the synthesis of $\beta$-amino carbonyl compounds via a three-component reaction involving aldehydes, ketones, and benzyl carbamates with good yields. However, this method has the disadvantage of longer reaction time [21].

As a part of our ongoing research in the use of solid support reagents [22], we wanted to expand the scope of iodine-alumina $\left(\mathrm{I}_{2}-\mathrm{Al}_{2} \mathrm{O}_{3}\right)$ as the catalyst for the synthesis of $\beta$-amino carbonyl compounds. We, therefore, report herein an efficient and time-saving one-pot protocol for 


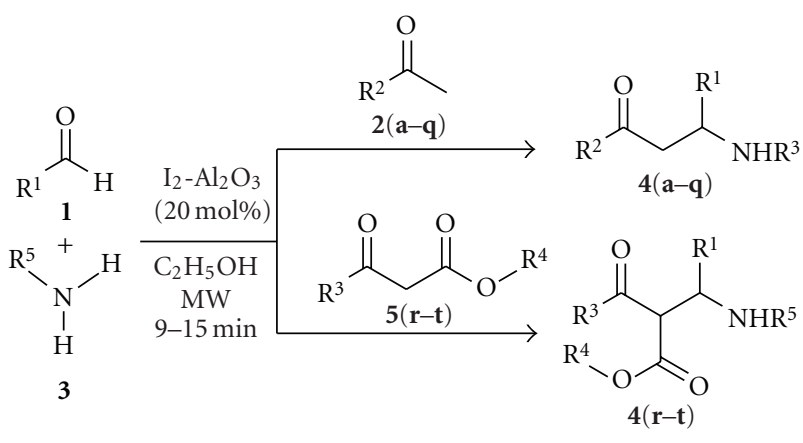

Scheme 1

the three-component condensation between an aldehyde, a substituted ketone, or 1,3-dicarbonyl compounds with substituted aniline using iodine-alumina $\left(\mathrm{I}_{2}-\mathrm{Al}_{2} \mathrm{O}_{3}\right)$ as a catalyst under microwave irradiation. This protocol was subsequently extended to the condensation with the less reactive methyl carbamate as a nitrogen source to give $\beta$ amino carbonyl compounds which can be easily deprotected to 1,3-amino alcohols or $\beta$-amino acids [20] (Scheme 1).

In the initial experiment, a mixture of 4-chlorobenzaldehyde (1a), acetophenone (2a), and 3-chloroaniline (3a) was refluxed in ethanol in presence of $\mathrm{I}_{2}-\mathrm{Al}_{2} \mathrm{O}_{3}(20 \mathrm{~mol} \%)$. On completion of the reaction ( $8 \mathrm{~h}$, monitored by TLC), workup and subsequent purification by column chromatography afforded the product $4 \mathrm{a}$ in $60 \%$ yield. The same reaction under microwave irradiation led to the formation of the desired $\beta$-amino carbonyl compound $4 \mathbf{a}(12 \mathrm{~min})$ in $92 \%$ yield. In a typical experiment, when a mixture of 3-chloroaniline (3a) (3.91 mmol), 4-chlorobenzaldehyde (1a) $(3.56 \mathrm{mmol})$, and acetophenone $(2 \mathrm{a})(3.91 \mathrm{mmol})$ was heated for $12 \mathrm{~min}$ in a microwave using $\mathrm{I}_{2}-\mathrm{Al}_{2} \mathrm{O}_{3}(0.71 \mathrm{mmol}$ of iodine adsorbed on $1.8 \mathrm{~g}$ of neutral alumina, that is, $20 \mathrm{~mol} \%$ arrived at after optimization is shown in Table 1) as a catalyst in dry ethanol at $100^{\circ} \mathrm{C}, 4 \mathbf{a}$ was obtained in $92 \%$ yield.

In another attempt, the same reaction mixture in neat condition under microwave irradiation in presence of $\mathrm{I}_{2}$ $\mathrm{Al}_{2} \mathrm{O}_{3}(20 \mathrm{~mol} \%)$ catalyst yielded only $46 \%$ of compound 4a. Later, the optimized reaction condition was extended for the condensation of methyl carbamate $(3 \mathbf{f})(3.91 \mathrm{mmol})$ with benzaldehyde (1e) $(3.55 \mathrm{mmol})$ and acetophenone (2a) (3.91 mmol) in presence of $\mathrm{I}_{2}-\mathrm{Al}_{2} \mathrm{O}_{3}(20 \mathrm{~mol} \%)$ in ethanol in a microwave. The reaction furnished the desired product 4f in $13 \mathrm{~min}$ with $75 \%$ yield. To study the generality of this methodology, reactions with a number of substituted aromatic aldehydes, substituted aromatic ketones, or 1,3dicarbonyl compounds and methyl carbamate or substituted anilines were carried out. Irrespective of the substitutions on the aromatic aldehydes, acetophenones or anilines by electron withdrawing or donating groups in either ortho, meta, or para position the reaction proceeded smoothly to give the corresponding products in good to excellent yields (Table 2). However, aldehyde having nitro group substitution failed to give the desired product, where as para-nitro acetophenone (2e) condenses readily with aniline (3b) and benzaldehyde (1e) to afford the product $4 \mathrm{e}$ (entry 5, Table 2). On the other hand, benzaldehyde (1e) when reacted with dicarbonyl compounds such as diethyl malonate (2t) and 3-chloroanilne (3a), compound $4 \mathbf{t}$ was obtained in 9 min with $89 \%$ yield (entry 20 , Table 2 ). All the $\beta$-amino carbonyl compounds (4a-4t) obtained after simple workup and purification by column chromatography was characterized by ${ }^{1} \mathrm{H},{ }^{13} \mathrm{C}$ NMR and IR analyses, and the results are summarized in Table 2.

It is to be noted that the effect of the $\mathrm{I}_{2}-\mathrm{Al}_{2} \mathrm{O}_{3}$ as compared to molecular iodine as a catalyst is more superior as is evident from the fact that, when the reaction is carried out with meta-substituted aldehyde, such as 3-methoxybenzaldehyde, it was found that $\mathrm{I}_{2}-\mathrm{Al}_{2} \mathrm{O}_{3}$ successfully gave the products $4 \mathrm{~h}, 4 \mathrm{l}, 4 \mathrm{r}$, and $4 \mathrm{~s}$ whereas the reported method using molecular iodine alone as a catalyst failed to yield the desired products [21]. Significantly, no iodination (for compound $\mathbf{4 q}$ ) was observed at the allylic double bond.

The formation of the $\beta$-amino carbonyl compounds may be explained as follows: alumina polarizes the iodine molecule and acts as an activating agent to produce a strongly electrophilic $\mathrm{I}^{+}$species. The $\mathrm{I}^{+}$species then catalyses in situ generation of acylimines which is subsequently attacked by the enolizable ketone to provide the desired compound.

\section{Conclusion}

In summary, we have established a convenient and environmentally benign protocol for the synthesis of $\beta$-amino carbonyl compounds by using $\mathrm{I}_{2}-\mathrm{Al}_{2} \mathrm{O}_{3}$ as a catalyst. In addition, this catalytic protocol has the advantages of mild reaction condition, simple workup procedure, and purification to afford the products in good yields. Due to its operational simplicity, this facile method is expected to have wider application for the preparation of $\beta$-amino carbonyl compounds.

\section{Experimental Section}

All commercially available chemicals and reagents were purchased from Aldrich and used without further purification. IR spectra were recorded on a Perkin-Elmer FT-IR instrument. The ${ }^{1} \mathrm{H}$ - and ${ }^{13} \mathrm{C}$-NMR spectrum were recorded on a Bruker Avance II 400 NMR machine. Unless otherwise specified, $\mathrm{CDCl}_{3}$ was used as solvent. Mass spectra were recorded with a Water ZQ-4000 equipped with ESI and APCI mass detector and CHN was done on Perkin-Elmer PE 2400 Series II. The $\mathrm{I}_{2}-\mathrm{Al}_{2} \mathrm{O}_{3}$ catalyst was prepared by the procedure reported in reference [24].

General Procedure $\mathbf{4}(\mathbf{a}-\mathbf{t})$. A prestirred mixture of aldehyde $(3.56 \mathrm{mmol})$, acetophenone, methyl acetoacetate, or diethyl malonate $(3.91 \mathrm{mmol})$ and methyl carbamate or aniline $(3.91 \mathrm{mmol})$ in dry ethanol $(3 \mathrm{~mL})$ was irradiated in a Chem Discover microwave reactor at $100^{\circ} \mathrm{C}$ (power $200 \mathrm{~W}$ ), for $10-15 \mathrm{~min}$, in the presence of $\mathrm{I}_{2}-\mathrm{Al}_{2} \mathrm{O}_{3}(20 \mathrm{~mol} \%)$. The completion of the reaction was monitored by thin layer chromatography. The reaction mixture was filtered through a bed of celite and washed the residue with ethyl acetate $(3 \times 5 \mathrm{~mL})$. The filtrate was then washed with aqueous 
TABLE 1: Optimization of the amount of $\mathrm{I}_{2}-\mathrm{Al}_{2} \mathrm{O}_{3}$ catalyst.

\begin{tabular}{|c|c|c|c|c|c|c|}
\hline Substrate $1 \mathrm{R}^{1}$ & Substrate $2 \mathrm{R}^{2}$ & Substrate $3 \mathrm{R}^{5}$ & $\mathrm{I}_{2}-\mathrm{Al}_{2} \mathrm{O}_{3}(\mathrm{~mol} \%)$ & Time (min) & Product 4 & Yield $^{\mathrm{a}} /(\%)$ \\
\hline $4-\mathrm{ClC}_{6} \mathrm{H}_{4}$ & $\mathrm{C}_{6} \mathrm{H}_{5}$ & $3-\mathrm{ClC}_{6} \mathrm{H}_{4}$ & 10 & 12 & $4 a$ & 57 \\
\hline $4-\mathrm{ClC}_{6} \mathrm{H}_{4}$ & $\mathrm{C}_{6} \mathrm{H}_{5}$ & $3-\mathrm{ClC}_{6} \mathrm{H}_{4}$ & 20 & 12 & $4 a$ & 92 \\
\hline $4-\mathrm{ClC}_{6} \mathrm{H}_{4}$ & $\mathrm{C}_{6} \mathrm{H}_{5}$ & $3-\mathrm{ClC}_{6} \mathrm{H}_{4}$ & 20 & 20 & $4 a$ & 90 \\
\hline $4-\mathrm{ClC}_{6} \mathrm{H}_{4}$ & $\mathrm{C}_{6} \mathrm{H}_{5}$ & $3-\mathrm{ClC}_{6} \mathrm{H}_{4}$ & 40 & 12 & $4 a$ & 91 \\
\hline
\end{tabular}

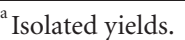

TABLE 2: $\mathrm{I}_{2}-\mathrm{Al}_{2} \mathrm{O}_{3}$ catalyzed multicomponent reaction for the preparation of $\beta$-amino carbonyl compounds.

\begin{tabular}{|c|c|c|c|c|c|c|}
\hline Entry & Substrate $1 \mathrm{R}^{1}$ & Substrate $2\left(\mathrm{R}^{2}\right) / \mathbf{5}\left(\mathrm{R}^{3}, \mathrm{R}^{4}\right)$ & Substrate $3 \mathrm{R}^{5}$ & Time $(\min )$ & Product $4^{\mathrm{b}}$ & Yield $^{\mathrm{a}} /(\%)^{\mathrm{c}}$ \\
\hline 1 & $4-\mathrm{ClC}_{6} \mathrm{H}_{4}$ & $\mathrm{R}^{2}=\mathrm{C}_{6} \mathrm{H}_{5}$ & $3-\mathrm{ClC}_{6} \mathrm{H}_{4}$ & 12 & $4 a$ & 92 \\
\hline 2 & $4-\mathrm{MeOC}_{6} \mathrm{H}_{4}$ & $\mathrm{R}^{2}=\mathrm{C}_{6} \mathrm{H}_{5}$ & $\mathrm{C}_{6} \mathrm{H}_{5}$ & 11 & $4 b$ & $90[23]$ \\
\hline 3 & $2-\mathrm{ClC}_{6} \mathrm{H}_{4}$ & $\mathrm{R}^{2}=\mathrm{C}_{6} \mathrm{H}_{5}$ & $4-\mathrm{NO}_{2} \mathrm{C}_{6} \mathrm{H}_{4}$ & 15 & $4 \mathrm{c}$ & 90 \\
\hline 4 & $3-\mathrm{BrC}_{6} \mathrm{H}_{4}$ & $\mathrm{R}^{2}=\mathrm{C}_{6} \mathrm{H}_{5}$ & $4-\mathrm{MeC}_{6} \mathrm{H}_{4}$ & 10 & $4 d$ & 86 \\
\hline 5 & $\mathrm{C}_{6} \mathrm{H}_{5}$ & $\mathrm{R}^{2}=4-\mathrm{NO}_{2} \mathrm{C}_{6} \mathrm{H}_{4}$ & $\begin{array}{r}\mathrm{C}_{6} \mathrm{H}_{5} \\
\mathrm{O}\end{array}$ & 12 & $4 e$ & 89 \\
\hline 6 & $\mathrm{C}_{6} \mathrm{H}_{5}$ & $\mathrm{R}^{2}=\mathrm{C}_{6} \mathrm{H}_{5}$ & $\mathrm{O}$ & 13 & $4 \mathrm{f}$ & 75 \\
\hline 7 & $4-\mathrm{MeC}_{6} \mathrm{H}_{4}$ & $\mathrm{R}^{2}=\mathrm{C}_{6} \mathrm{H}_{5}$ & $\mathrm{O}$ & 12 & $4 g$ & 65 \\
\hline 8 & $3-\mathrm{MeOC}_{6} \mathrm{H}_{4}$ & $\mathrm{R}^{2}=\mathrm{C}_{6} \mathrm{H}_{5}$ & $\mathrm{O}$ & 11 & $4 h$ & 75 \\
\hline 9 & $4-\mathrm{MeOC}_{6} \mathrm{H}_{4}$ & $\mathrm{R}^{2}=\mathrm{C}_{6} \mathrm{H}_{5}$ & $\mathrm{O}$ & 12 & $4 \mathbf{i}$ & 75 \\
\hline 10 & $4-\mathrm{ClC}_{6} \mathrm{H}_{4}$ & $\mathrm{R}^{2}=\mathrm{C}_{6} \mathrm{H}_{5}$ & $\mathrm{O}$ & 13 & $4 j$ & 70 \\
\hline 11 & $4-\mathrm{OHC}_{6} \mathrm{H}_{4}$ & $\mathrm{R}^{2}=\mathrm{C}_{6} \mathrm{H}_{5}$ & $\mathrm{O}$ & 13 & $4 k$ & 96 \\
\hline 12 & $3,4-(\mathrm{MeO})_{2} \mathrm{C}_{6} \mathrm{H}_{3}$ & $\mathrm{R}^{2}=\mathrm{C}_{6} \mathrm{H}_{5}$ & $\mathrm{O}$ & 10 & 41 & 73 \\
\hline 13 & $4-\mathrm{ClC}_{6} \mathrm{H}_{4}$ & $\mathrm{R}^{2}=2-\mathrm{MeC}_{6} \mathrm{H}_{4}$ & O & 15 & $4 \mathrm{~m}$ & 70 \\
\hline 14 & $4-\mathrm{ClC}_{6} \mathrm{H}_{4}$ & $\mathrm{R}^{2}=4-\mathrm{MeOC}_{6} \mathrm{H}_{4}$ & $\mathrm{O}$ & 10 & $4 n$ & 65 \\
\hline 15 & $4-\mathrm{ClC}_{6} \mathrm{H}_{4}$ & $\mathrm{R}^{2}=4-\mathrm{BrC}_{6} \mathrm{H}_{4}$ & $\mathrm{O}$ & 14 & 40 & 72 \\
\hline 16 & $4-\mathrm{ClC}_{6} \mathrm{H}_{4}$ & $\mathrm{R}^{2}=4-\mathrm{OHC}_{6} \mathrm{H}_{4}$ & & 15 & $4 p$ & 69 \\
\hline 17 & & $\mathrm{R}^{2}=\mathrm{C}_{6} \mathrm{H}_{5}$ & & 14 & $4 q$ & 68 \\
\hline 18 & $3,4-(\mathrm{MeO})_{2} \mathrm{C}_{6} \mathrm{H}_{3}$ & $\begin{array}{l}\mathrm{R}^{3}=\mathrm{Me} \\
\mathrm{R}^{4}=\mathrm{Me}\end{array}$ & & 9 & $4 r$ & 75 \\
\hline 19 & $3,4,5-(\mathrm{MeO}){ }_{3} \mathrm{C}_{6} \mathrm{H}_{2}$ & $\begin{array}{l}\mathrm{R}^{3}=\mathrm{Me} \\
\mathrm{R}^{4}=\mathrm{Me}\end{array}$ & & 15 & $4 s$ & 67 \\
\hline 20 & $\mathrm{C}_{6} \mathrm{H}_{5}$ & $\begin{array}{c}\mathrm{R}^{3}=\mathrm{OEt} \\
\mathrm{R}^{4}=\mathrm{Et}\end{array}$ & $3-\mathrm{ClC}_{6} \mathrm{H}_{4}$ & 9 & $4 \mathrm{t}$ & 89 \\
\hline
\end{tabular}

${ }^{\mathrm{a}}$ Isolated yields. ${ }^{\mathrm{b}}$ Products were characterized by ${ }^{1} \mathrm{H},{ }^{13} \mathrm{C}$ NMR and IR analyses. ${ }^{\mathrm{c}}$ Reference. 
sodium thiosulfate (5\%) followed by brine. The organic layer was dried over anhydrous $\mathrm{Na}_{2} \mathrm{SO}_{4}$ and concentrated in vacuo to give the crude mass. The crude compound was then purified by silica gel column chromatography, using ethyl acetate and hexane as eluents, to afford the $\beta$-amino carbonyl compounds in pure form.

3-(4-Chlorophenyl)-3-((3-chlorophenyl)amino)-1-phenylpropan-1-one (4a, Table 2). Off white solid; mp $98-100{ }^{\circ} \mathrm{C} ;{ }^{1} \mathrm{H}$ NMR (400 MHz, DMSO-d $)_{6}: \delta 3.07(1 \mathrm{H}, \mathrm{dd}, J=4,17.2 \mathrm{~Hz}$, $\left.\mathrm{CH}_{2}\right), 3.41\left(1 \mathrm{H}, \mathrm{dd}, J=8.8,17.2 \mathrm{~Hz}, \mathrm{CH}_{2}\right), 4.75-4.80(1 \mathrm{H}$, $\mathrm{m}, \mathrm{CH}), 6.23(2 \mathrm{H}, \mathrm{t}, J=6 \mathrm{~Hz}, \operatorname{Ar}-\mathrm{H}), 6.27(1 \mathrm{H}, \mathrm{d}, J=$ $1.2 \mathrm{~Hz}, \mathrm{NH}), 6.38(1 \mathrm{H}, \mathrm{d}, J=7.2 \mathrm{~Hz}, \mathrm{Ar}-\mathrm{H}), 6.75(1 \mathrm{H}, \mathrm{t}, J$ $=8 \mathrm{~Hz}, \mathrm{Ar}-\mathrm{H}), 7.13(2 \mathrm{H}, \mathrm{d}, J=8.4 \mathrm{~Hz}, \mathrm{Ar}-\mathrm{H}), 7.19-7.40(5 \mathrm{H}$, $\mathrm{m}, \operatorname{Ar}-\mathrm{H}), 7.73(2 \mathrm{H}, \mathrm{d}, J=7.2 \mathrm{~Hz}, \mathrm{Ar}-\mathrm{H}) \mathrm{ppm} ;{ }^{13} \mathrm{C} \mathrm{NMR}$ $\left(100 \mathrm{MHz}, \mathrm{DMSO}-d_{6}\right): \delta 46.1,51.9,111.4,111.9,115.4$, $128.0,128.3,128.5,128.7,130.3,131.4,133.4,136.5,142.5$, 149.1, 196.7 ppm; IR. $\nu_{\max }(\mathrm{KBr}): 3398,3062,2925,2900$, 2853, 1676, 1599, 1518, 1489, 1293, $1089 \mathrm{~cm}^{-1}$; Elemental analysis for $\mathrm{C}_{21} \mathrm{H}_{17} \mathrm{C}_{22} \mathrm{NO}$ Calcd. C, 68.12; H, 4.63; N, 3.78; Found: C, 67.96; H, 4.70; N, 3.68.

3-(4-Methoxyphenyl)-1-phenyl-3-(phenylamino)propan-1-one (4b, Table 2). The spectroscopic data is in full agreement with the literature data [23].

3-(2-Chlorophenyl)-3-((4-nitrophenyl)amino)-1-phenylpropan -1-one (4c, Table 2). Yellow solid; mp $135-137^{\circ} \mathrm{C} ;{ }^{1} \mathrm{H}$ NMR $\left(400 \mathrm{MHz} \mathrm{CDCl}_{3}\right): \delta 3.49\left(2 \mathrm{H}, \mathrm{d}, J=6.2 \mathrm{~Hz}, \mathrm{CH}_{2}\right), 5.38$ $(1 \mathrm{H}, \mathrm{m}, \mathrm{CH}), 5.48(1 \mathrm{H}, \mathrm{d}, J=6.0 \mathrm{~Hz}, \mathrm{NH}), 6.40(2 \mathrm{H}, \mathrm{d}, J$ = 9.2 Hz, Ar-H), 7.20-7.50 (7H, m, Ar-H), $7.67(2 \mathrm{H}, \mathrm{d}, J=$ $7.2 \mathrm{~Hz}, \mathrm{Ar}-\mathrm{H}), 7.91(2 \mathrm{H}, \mathrm{d}, J=9.0 \mathrm{~Hz}, \mathrm{Ar}-\mathrm{H}) \mathrm{ppm} ;{ }^{13} \mathrm{C} \mathrm{NMR}$ $\left(100 \mathrm{MHz} \mathrm{CDCl}_{3}\right): \delta 42.8,51.6,112.4,126.2,127.7,127.8$, $128.4,128.9,129.2,130.2,132.4,134.0,136.2,137.7,138.7$, 151.6, 198.2 ppm; IR $\nu_{\max }(\mathrm{KBr}): 3369,3062,2925,2854$, 1691, 1598, 1532, 1503, 1471, 1449, 1308, 1291, $1113 \mathrm{~cm}^{-1}$; Elemental anlaysis for $\mathrm{C}_{21} \mathrm{H}_{17} \mathrm{ClN}_{2} \mathrm{O}_{3}$ Calcd. C, 66.23; $\mathrm{H}$, 4.50; N, 7.36; Found: C 66.45; H 4.43; N 7.41.

3-(3-Bromophenyl)-1-phenyl-3-(p-tolylamino)propan-1-one (4d, Table 2). White solid; mp $107-111^{\circ} \mathrm{C} ;{ }^{1} \mathrm{H}$ NMR $(400 \mathrm{MHz}$, $\left.\mathrm{CDCl}_{3}\right): \delta 2.14\left(3 \mathrm{H}, \mathrm{s}, \mathrm{CH}_{3}\right), 3.39(2 \mathrm{H}, \mathrm{dd}, J=6,18 \mathrm{~Hz}$, $\left.\mathrm{CH}_{2}\right), 3.61(1 \mathrm{H}, \mathrm{d}, J=5.6 \mathrm{~Hz}, \mathrm{NH}), 4.87(1 \mathrm{H}, \mathrm{t}, J=6.4 \mathrm{~Hz}$, $\mathrm{CH}), 6.61(2 \mathrm{H}, \mathrm{d}, J=7.6 \mathrm{~Hz}, \mathrm{Ar}-\mathrm{H}), 6.88(2 \mathrm{H}, \mathrm{d}, J=8 \mathrm{~Hz}$, Ar-H), 7.02-7.52 (6H, m, Ar-H), 7.78 (2H, d, J = 7.6 Hz, Ar$\mathrm{H}), 7.87(1 \mathrm{H}, \mathrm{d}, J=8.4 \mathrm{~Hz}, \mathrm{Ar}-\mathrm{H}) \mathrm{ppm} ;{ }^{13} \mathrm{C} \mathrm{NMR}(100 \mathrm{MHz}$, $\left.\mathrm{CDCl}_{3}\right): \delta 20.6,44.8,59.5,117.0,122.9,127.0,128.1,128.2$, $128.7,128.8,129.5,129,9,130.4,130.4,131.1,131.3,131.6$, 133.6, 136.3, 197.0 ppm; IR. $v_{\max }(\mathrm{KBr}): 3403,2924,2854$, $1678,1620,1597,1523,1366,1289 \mathrm{~cm}^{-1}$; Elemental analysis for $\mathrm{C}_{22} \mathrm{H}_{20}$ BrNO Calcd. C, 67.01; H, 5.11; N, 3.55; Found C, 67.23; H, 5.55; N, 3.24.

1-(4-nitrophenyl)-3-phenyl-3-(phenylamino)propan-1-one (4e, Table 2). White Solid; mp $145-147^{\circ} \mathrm{C} ;{ }^{1} \mathrm{H}$ NMR $(400 \mathrm{MHz}$, $\left.\mathrm{CDCl}_{3}\right): \delta 3.40-3.58\left(2 \mathrm{H}, \mathrm{m}, \mathrm{CH}_{2}\right), 5.16(1 \mathrm{H}, \mathrm{t}, \mathrm{CH}), 6.69$ $(2 \mathrm{H}, \mathrm{d}, J=6.4 \mathrm{~Hz}, \mathrm{Ar}-\mathrm{H}), 6.63-6.70$ (2H, m, Ar-H). 6.796.89 (1H, m, Ar-H), 7.14 (2H, d, J = 7.6 Hz, Ar-H), 7.26-7.29
(1H, m, Ar-H), 7.31-7.35 (2H, m, Ar-H), $7.66(2 \mathrm{H}, \mathrm{d}, J=$ $6.4 \mathrm{~Hz}, \mathrm{Ar}-\mathrm{H}) ; 7.90(2 \mathrm{H}, \mathrm{d}, J=7.2 \mathrm{~Hz}, \mathrm{Ar}-\mathrm{H}) \mathrm{ppm} ;{ }^{13} \mathrm{C} \mathrm{NMR}$ $\left(100 \mathrm{MHz}, \mathrm{CDCl}_{3}\right): \delta 46.0,53.5,110.0,114.8,122.9,124.0$, $126.1,128.0,128.3,128.9,142.7,141.3,151.1,196.5$ ppm; IR. $\nu_{\max }(\mathrm{KBr}): 3400,3099,3064,2985,2925,2872,1678$, $1600,1538,1352,1214,1204 \mathrm{~cm}^{-1}$; Elemental analysis for $\mathrm{C}_{21} \mathrm{H}_{18} \mathrm{~N}_{2} \mathrm{O}_{3}$ : Calcd. C, 72.82; H, 5.24; N, 8.09; Found: C, 72.96; H, 5.34; N, 8.11.

Methyl (3-oxo-1,3-diphenylpropyl)carbamate (4f, Table 2). Off white solid; mp $115-120^{\circ} \mathrm{C}$; ${ }^{1} \mathrm{H}$ NMR $(400 \mathrm{MHz}$, $\left.\mathrm{CDCl}_{3}\right): \delta 3.43-3.49\left(2 \mathrm{H}, \mathrm{m}, \mathrm{CH}_{2}\right), 3.66\left(3 \mathrm{H}, \mathrm{s}, \mathrm{COOCH}_{3}\right)$, 5.29-5.31 (1H, m, CH), $5.77(1 \mathrm{H}, \mathrm{s}, \mathrm{NH}), 7.22-7.46(7 \mathrm{H}, \mathrm{m}$, Ar-H), $7.56(1 \mathrm{H}, \mathrm{t}, J=7.2 \mathrm{~Hz}, \mathrm{Ar}-\mathrm{H}), 7.90(2 \mathrm{H}, \mathrm{d}, J=7.6 \mathrm{~Hz}$, Ar-H) ppm; $\left.{ }^{13} \mathrm{C} \mathrm{NMR} \mathrm{(100} \mathrm{MHz,} \mathrm{CDCl}_{3}\right): \delta 30.9,43.9,51.7$, 52.2, 126.3, 127.4, 128.1, 128.6, 128.7, 133.4, 136.6, 141.4, $156.4,197.8$ ppm; IR. $v_{\max }(\mathrm{KBr}): 3369,2955,2924,2853$, 1733, 1683, 1521, 1294, $1030 \mathrm{~cm}^{-1}$; Elemental analysis for $\mathrm{C}_{17} \mathrm{H}_{17} \mathrm{NO}_{3}$ : Calcd. C, 72.07; H, 6.05; N, 4.94; Found C, 72.27; H, 6.17; N, 4.86 .

Methyl (3-oxo-3-phenyl-1-(p-tolyl)propyl)carbamate (4g, Table 2). Brownish solid; mp $90-94{ }^{\circ} \mathrm{C} ;{ }^{1} \mathrm{H}$ NMR $(400 \mathrm{MHz}$, $\left.\mathrm{CDCl}_{3}\right): \delta 2.30\left(3 \mathrm{H}, \mathrm{s}, \mathrm{CH}_{3}\right), 3.44(2 \mathrm{H}, \mathrm{dd}, J=6,16.8 \mathrm{~Hz}$, $\left.\mathrm{CH}_{2}\right), 3.66\left(3 \mathrm{H}, \mathrm{s}, \mathrm{COOCH}_{3}\right), 5.25-5.29(1 \mathrm{H}, \mathrm{m}, \mathrm{CH}), 5.72$ $(1 \mathrm{H}, \mathrm{s}, \mathrm{NH}), 7.12(2 \mathrm{H}, \mathrm{d}, J=7.6 \mathrm{~Hz}, \mathrm{Ar}-\mathrm{H}), 7.24(2 \mathrm{H}, \mathrm{d}$, $J=8 \mathrm{~Hz}, \mathrm{Ar}-\mathrm{H}), 7.45(1 \mathrm{H}, \mathrm{t}, J=7.6 \mathrm{~Hz}, \mathrm{Ar}-\mathrm{H}), 7.55(2 \mathrm{H}$, $\mathrm{d}, J=7.6 \mathrm{~Hz}, \mathrm{Ar}-\mathrm{H}), 7.91(2 \mathrm{H}, \mathrm{d}, J=7.6 \mathrm{~Hz}, \mathrm{Ar}-\mathrm{H}) \mathrm{ppm}$; ${ }^{13} \mathrm{C} \mathrm{NMR}\left(100 \mathrm{MHz}, \mathrm{CDCl}_{3}\right): \delta 21.0,44.0,51.5,52.2,126.3$, $128.1,128.7,129.3,133.4,136.6,137.1,138.3,156.3$ ppm; IR. $\nu_{\max }(\mathrm{KBr}): 3305,3068,3013,2994,2947,2923,2909$, 1714, 1690, 1551, 1276, 1050, $763 \mathrm{~cm}^{-1}$; Elemental analysis for $\mathrm{C}_{18} \mathrm{H}_{19} \mathrm{NO}_{3}$ Calcd. C, 72.71; H, 6.44; N, 4.71; Found C, $72.91 ; \mathrm{H}, 6.36 ; \mathrm{N}, 4.68$.

Methyl (1-(3-methoxyphenyl)-3-oxo-3-phenylpropyl)carbamate (4h, Table 2). Yellowish solid; mp $100-104^{\circ} \mathrm{C} ;{ }^{1} \mathrm{H}$ NMR $\left(400 \mathrm{MHz}, \mathrm{CDCl}_{3}\right): \delta 3.43\left(2 \mathrm{H}, \mathrm{dd}, J=4.8,16.4 \mathrm{~Hz}, \mathrm{CH}_{2}\right)$, $3.68\left(3 \mathrm{H}, \mathrm{s}, \mathrm{COOCH}_{3}\right), 3.78\left(3 \mathrm{H}, \mathrm{s}, \mathrm{COCH}_{3}\right), 5.25-5.30(1 \mathrm{H}$, $\mathrm{m}, \mathrm{CH}), 5.88(1 \mathrm{H}, \mathrm{d}, J=6.4 \mathrm{~Hz}, \mathrm{NH}), 6.78(2 \mathrm{H}, \mathrm{d}, J=8 \mathrm{~Hz}$, Ar-H), 6.90-7.27 (2H, m, Ar-H), 7.44 (2H, t, J = 7.6 Hz, Ar$\mathrm{H}), 7.56(1 \mathrm{H}, \mathrm{t}, J=7.2 \mathrm{~Hz}, \mathrm{Ar}-\mathrm{H}), 7.90(2 \mathrm{H}, \mathrm{d}, J=7.6 \mathrm{~Hz}$, $\mathrm{Ar}-\mathrm{H}) \mathrm{ppm} ;{ }^{13} \mathrm{C} \mathrm{NMR}\left(100 \mathrm{MHz}, \mathrm{CDCl}_{3}\right): \delta 43.9,51.7,52.3$, 55.2, 112.3, 112.6, 118.5, 128.1, 128.7, 129.7, 133.4, 136.6, 156.4, 157.6, 159.7 ppm; IR. $\nu_{\max }(\mathrm{KBr}): 3362,3080,3047$, 3007, 2962, 2925, 2852, 1728, 1683, 1598, 1518, 1491, 1295, $1167,1022 \mathrm{~cm}^{-1}$; Elemental analysis for $\mathrm{C}_{18} \mathrm{H}_{19} \mathrm{NO}_{4}$ Calcd. C, 68.99; H, 6.11; N, 4.47; Found C, 69.12; H, 6.15; N, 4.31.

Methyl (1-(4-methoxyphenyl)-3-oxo-3-phenylpropyl)carbamate (4i, Table 2). Yellowish solid; $\mathrm{mp} 145-148^{\circ} \mathrm{C} ;{ }^{1} \mathrm{H}$ NMR $\left(400 \mathrm{MHz}, \mathrm{CDCl}_{3}\right): \delta 3.43\left(2 \mathrm{H}, \mathrm{dd}, J=6.4,16.8 \mathrm{~Hz}, \mathrm{CH}_{2}\right)$, $3.66\left(3 \mathrm{H}, \mathrm{s}, \mathrm{COOCH}_{3}\right), 3.77\left(3 \mathrm{H}, \mathrm{s}, \mathrm{COCH}_{3}\right), 5.24-5.25(1 \mathrm{H}$, $\mathrm{m}, \mathrm{CH}), 5.66(1 \mathrm{H}, \mathrm{s}, \mathrm{NH}), 6.84(2 \mathrm{H}, \mathrm{d}, J=8.8 \mathrm{~Hz}, \mathrm{Ar}-\mathrm{H})$, 7.26-7.28 (2H, m, Ar-H), 7.43-7.46 (2H, m, Ar-H), 7.55 $(1 \mathrm{H}, \mathrm{t}, J=7.6 \mathrm{~Hz}, \mathrm{Ar}-\mathrm{H}), 7.90(2 \mathrm{H}, \mathrm{d}, J=9.6 \mathrm{~Hz}, \mathrm{Ar}-\mathrm{H}) \mathrm{ppm}$; ${ }^{13} \mathrm{C} \mathrm{NMR}\left(100 \mathrm{MHz}, \mathrm{CDCl}_{3}\right): \delta 51.4,52.2,55.3,114.0,127.6$, $128.1,128.7,133.4,136.7,156.3,158.9$ ppm; IR. $\nu_{\max }(\mathrm{KBr})$ : 
3296, 3074, 3022, 2994, 2966, 2948, 2906, 2844, 1709, 1687, 1555, 1513, 1280, 1251, 1048, $762 \mathrm{~cm}^{-1}$; Elemental Analysis for $\mathrm{C}_{18} \mathrm{H}_{19} \mathrm{NO}_{4}$ Calcd. C, 68.99; H, 6.11; N, 4.47; Found C, 68.89; H, 6.16; N, 4.39.

Methyl (1-(4-chlorophenyl)-3-oxo-3-phenylpropyl)carbamate (4j, Table 2). Off white solid; mp ${ }^{109-112}{ }^{\circ} \mathrm{C} ;{ }^{1} \mathrm{H}$ NMR $\left(400 \mathrm{MHz} \mathrm{CDCl}_{3}\right): \delta 3.45-3.47\left(2 \mathrm{H}, \mathrm{m}, \mathrm{CH}_{2}\right), 3.66(3 \mathrm{H}, \mathrm{s}$, $\left.\mathrm{COOCH}_{3}\right), 5.25-5.29(1 \mathrm{H}, \mathrm{m}, \mathrm{CH}), 5.86(1 \mathrm{H}, \mathrm{s}, \mathrm{NH}), 7.26-$ 7.47 (6H, m. Ar-H), 7.58 (1H. t, $J=7.6 \mathrm{~Hz}, \mathrm{Ar}-\mathrm{H}), 7.89(2 \mathrm{H}$, d, $J=7.6 \mathrm{~Hz}, \mathrm{Ar}-\mathrm{H})$ ppm; IR. $\nu_{\max }(\mathrm{KBr}): 3311,3061,2953$, 2853, 1693, 1545, 1274, 1052, $761 \mathrm{~cm}^{-1}$; Elemental Analysis for $\mathrm{C}_{17} \mathrm{H}_{16} \mathrm{ClNO}_{3}$ Calcd. C, 64.26; H, 5.08; N, 4.41; Found C, 64.29; H, 5.12; N, 4.53.

Methyl (1-(4-hydroxyphenyl)-3-oxo-3-phenylpropyl)carbamate (4k, Table 2). Brownish liquid; ${ }^{1} \mathrm{H}$ NMR (400 $\left.\mathrm{MHz}, \mathrm{CDCl}_{3}\right)$ : $\delta 3.40\left(2 \mathrm{H}, \mathrm{dd}, J=6,16.8 \mathrm{~Hz}, \mathrm{CH}_{2}\right), 3.67\left(3 \mathrm{H}, \mathrm{s}, \mathrm{COOCH}_{3}\right)$, 5.20-5.21 (1H, m, CH), $5.78(1 \mathrm{H}, \mathrm{s}, \mathrm{NH}), 6.69(2 \mathrm{H}, \mathrm{d}, J=$ $8 \mathrm{~Hz}, \mathrm{Ar}-\mathrm{H}), 7.15(2 \mathrm{H}, \mathrm{d}, J=8 \mathrm{~Hz}, \mathrm{Ar}-\mathrm{H}), 7.42-7.46(2 \mathrm{H}, \mathrm{m}$, Ar-H), $7.56(1 \mathrm{H}, \mathrm{t}, J=7.2 \mathrm{~Hz}, \mathrm{Ar}-\mathrm{H}), 7.90(2 \mathrm{H}, \mathrm{d}, J=7.6 \mathrm{~Hz}$, Ar-H) ppm; ${ }^{13} \mathrm{C} \mathrm{NMR}\left(100 \mathrm{MHz}, \mathrm{CDCl}_{3}\right): \delta 44.1,51.5$, 52.4, 115.5, 115.9, 127.6, 128.2, 128.7, 133.5, 136.5, 155.4, 156.6 ppm; IR. $\nu_{\max }(\mathrm{KBr}): 3438,3019,1716,1600,1514$, $1215,1018,768 \mathrm{~cm}^{-1}$; Elemental Analysis for $\mathrm{C}_{17} \mathrm{H}_{17} \mathrm{NO}_{4}$ Calcd. C, 68.21; H, 5.72; N, 4.68; Found C, 68.36; H, 5.63; $\mathrm{N}, 4.72$.

Methyl (1-(3,4-dimethoxyphenyl)-3-oxo-3-phenylpropyl)carbamate (4l, Table 2). White solid; mp $117-120^{\circ} \mathrm{C} ;{ }^{1} \mathrm{H}$ NMR $\left(400 \mathrm{MHz}_{\mathrm{CDCl}}\right): \delta 3.42\left(2 \mathrm{H}, \mathrm{dd}, J=6,16.4 \mathrm{~Hz}, \mathrm{CH}_{2}\right)$, $3.65\left(3 \mathrm{H}, \mathrm{s}, \mathrm{COOCH}_{3}\right), 3.83\left(3 \mathrm{H}, \mathrm{s}, \mathrm{COCH}_{3}\right), 3.84(3 \mathrm{H}, \mathrm{s}$, $\left.\mathrm{COCH}_{3}\right), 5.25(1 \mathrm{H}, \mathrm{br}, \mathrm{CH}), 5.79(1 \mathrm{H}, \mathrm{s}, \mathrm{NH}), 6.79(1 \mathrm{H}$, d, $J=8.4 \mathrm{~Hz}, \mathrm{Ar}-\mathrm{H}), 6.88(1 \mathrm{H}, \mathrm{d}, J=4.8 \mathrm{~Hz}, \mathrm{Ar}-\mathrm{H}), 7.42-$ $7.46(3 \mathrm{H}, \mathrm{m}, \mathrm{Ar}-\mathrm{H}), 7.56(1 \mathrm{H}, \mathrm{t}, J=7.2 \mathrm{~Hz}, \mathrm{Ar}-\mathrm{H}), 7.90(2 \mathrm{H}$, $\mathrm{d}, J=7.6 \mathrm{~Hz}, \mathrm{Ar}-\mathrm{H}) \mathrm{ppm} ;{ }^{13} \mathrm{C} \mathrm{NMR}\left(100 \mathrm{MHz}, \mathrm{CDCl}_{3}\right): \delta$ 44.1, 51.7, 52.2, 55.9, 110.0, 111.1, 118.3, 128.1, 128.7, 133.4, 136.7, 148.3, 149.0, 156.4, 198.1 ppm; IR. $\nu_{\max }(\mathrm{KBr}): 3379$, 3078, 2994, 2959, 2924, 2852, 1728, 1676, 1592, 1535, 1522, 1452, 1295, 1264, 1025, $757 \mathrm{~cm}^{-1}$; Elemental Analysis for $\mathrm{C}_{19} \mathrm{H}_{21} \mathrm{NO}_{5}$ Calcd. C, 66.46; H, 6.16; N, 4.08; Found C, 66.59; $\mathrm{H}, 6.23 ; \mathrm{N}, 4.16$.

Methyl (1-(4-chlorophenyl)-3-oxo-3-(o-tolyl)propyl)carbamate (4m, Table 2). Off white solid; mp $110-114^{\circ} \mathrm{C} ;{ }^{1} \mathrm{H}$ NMR $\left(400 \mathrm{MHz}_{\mathrm{CDCl}}\right): \delta 2.36\left(3 \mathrm{H}, \mathrm{s}, \mathrm{CH}_{3}\right), 3.36(2 \mathrm{H}, \mathrm{dd}, J=5.6$, $\left.16.4 \mathrm{~Hz}, \mathrm{CH}_{2}\right), 3.67\left(3 \mathrm{H}, \mathrm{s}, \mathrm{COOCH}_{3}\right), 5.20-5.22(1 \mathrm{H}, \mathrm{m}$, $\mathrm{CH}), 5.84(1 \mathrm{H}, \mathrm{s}, \mathrm{NH}), 7.21-7.39(7 \mathrm{H}, \mathrm{m}, \mathrm{Ar}-\mathrm{H}), 7.53(1 \mathrm{H}$, d, $J=7.6 \mathrm{~Hz}, \mathrm{Ar}-\mathrm{H}) \mathrm{ppm} ;{ }^{13} \mathrm{C} \mathrm{NMR}\left(100 \mathrm{MHz}, \mathrm{CDCl}_{3}\right): \delta$ 21.2, 31.0, 51.4, 52.3, 125.8, 127.8, 128.5, 128.8, 131.9, 132.2, $133.3,137.2,138.5,139.9,154.5,194.3$ ppm; IR. $v_{\max }(\mathrm{KBr})$ : 3319, 3062, 3027, 2951, 2930, 2853, 1690, 1678, 1547, 1491, $1345,1273,1053 \mathrm{~cm}^{-1}$; Elemental Analysis for $\mathrm{C}_{18} \mathrm{H}_{18} \mathrm{ClNO}_{3}$ Calcd. C, 65.16; H, 5.47; N, 4.22; Found C, 65.21; H, 5.53; N, 4.26 .

Methyl (1-(4-chlorophenyl)-3-(4-methoxyphenyl)-3-oxopropyl) carbamate (4n, Table 2). White solid; mp $120-124^{\circ} \mathrm{C} ;{ }^{1} \mathrm{H}$
NMR $\left(400 \mathrm{MHz}, \mathrm{CDCl}_{3}\right): \delta 3.36(2 \mathrm{H}, \mathrm{dd}, J=6,16.8 \mathrm{~Hz}$, $\left.\mathrm{CH}_{2}\right), 3.66\left(3 \mathrm{H}, \mathrm{s}, \mathrm{COOCH}_{3}\right), 3.86\left(3 \mathrm{H}, \mathrm{s}, \mathrm{COCH}_{3}\right), 5.21-$ $5.26(1 \mathrm{H}, \mathrm{m}, \mathrm{CH}), 5.94(1 \mathrm{H}, \mathrm{s}, \mathrm{NH}), 6.91(2 \mathrm{H}, \mathrm{d}, J=8.8 \mathrm{~Hz}$, Ar-H), 7.25-7.30 (4H, m, Ar-H), $7.86(2 \mathrm{H}, \mathrm{d}, J=9.2 \mathrm{~Hz}, \mathrm{Ar}-$ $\mathrm{H}) \mathrm{ppm} ;{ }^{13} \mathrm{C} \mathrm{NMR}\left(100 \mathrm{MHz}, \mathrm{CDCl}_{3}\right): \delta$ 43.2, 51.3, 52.3, 55.5, 113.9, 127.8, 128.7, 129.6, 130.4, 133.1, 140.1, 156.4, 163.9 ppm; IR. $\nu_{\max }(\mathrm{KBr}): 3307,3061,3008,2951,2840$, 1685, 1668, 1604, 1540, 1421, 1260, $1045 \mathrm{~cm}^{-1}$; Elemental Analysis for $\mathrm{C}_{18} \mathrm{H}_{18} \mathrm{ClNO}_{4}$ Calcd. C, 62.16; H, 5.22; N, 4.03; Found C, 62.13; H, 5.24; N, 4.11 .

Methyl (3-(4-bromophenyl)-1-(4-chlorophenyl)-3-oxopropyl) carbamate (4o, Table 2). White solid; $\mathrm{mp} 87-91^{\circ} \mathrm{C} ;{ }^{1} \mathrm{H}$ NMR $\left(400 \mathrm{MHz} \mathrm{CDCl}_{3}\right): \delta 3.4\left(2 \mathrm{H}, \mathrm{dd}, J=6,17.2 \mathrm{~Hz}, \mathrm{CH}_{2}\right), 3.67$ $\left(3 \mathrm{H}, \mathrm{s}, \mathrm{COOCH}_{3}\right), 5.23-5.28(1 \mathrm{H}, \mathrm{m}, \mathrm{CH}), 5.75(1 \mathrm{H}, \mathrm{s}, \mathrm{NH})$, 7.23-7.29 (4H, m, Ar-H), $7.59(2 \mathrm{H}, \mathrm{d}, J=8.8 \mathrm{~Hz}, \mathrm{Ar}-\mathrm{H})$, $7.75(2 \mathrm{H}, \mathrm{d}, J=8.8 \mathrm{~Hz}, \mathrm{Ar}-\mathrm{H}) \mathrm{ppm} ;{ }^{13} \mathrm{C} \mathrm{NMR}(100 \mathrm{MHz}$, $\left.\mathrm{CDCl}_{3}\right): \delta 45.7,51.1,52.3,127.8,128.8,128.9,129.6,130.9$, $132.1,133.3,135.1,156.3,190.9$ ppm; IR. $\nu_{\max }(\mathrm{KBr}): 3319$, 3072, 2926, 2853, 1688, 1586, 1540, 1264, 1199, $1012 \mathrm{~cm}^{-1}$; Elemental Analysis for $\mathrm{C}_{17} \mathrm{H}_{15} \mathrm{BrClNO}_{3}$ Calcd. C, 51.47; H, 3.81; N, 3.53; Found C, 51.39; H, 3.76; N, 3.58.

Methyl (1-(4-chlorophenyl)-3-(4-hydroxyphenyl)-3-oxopropyl) carbamate (4p, Table 2). Off white solid; mp $100-104^{\circ} \mathrm{C}$; ${ }^{1} \mathrm{H}$ NMR $\left(400 \mathrm{MHz}, \mathrm{CDCl}_{3}\right): \delta 3.36-3.51\left(2 \mathrm{H}, \mathrm{m}, \mathrm{CH}_{2}\right)$, $3.65\left(3 \mathrm{H}, \mathrm{s}, \mathrm{COOCH}_{3}\right), 5.23(1 \mathrm{H}, \mathrm{s}, \mathrm{CH}), 6.85(2 \mathrm{H}, \mathrm{d}, J=$ 8.4 Hz, Ar-H), 7.27-7.30 (2H, m, Ar-H), $7.52(2 \mathrm{H}, \mathrm{d}, J=$ $8 \mathrm{~Hz}, \mathrm{Ar}-\mathrm{H}), 7.78(2 \mathrm{H}, \mathrm{d}, J=8.4 \mathrm{~Hz}, \mathrm{Ar}-\mathrm{H}) \mathrm{ppm} ;{ }^{13} \mathrm{C}$ NMR $\left(100 \mathrm{MHz} \mathrm{CDCl}_{3}\right): \delta 52.4,65.9,115.5,127.8,128.7,129.5$, $130.7,130.9,132.2,133.1,156.6,161.3,190.9$ ppm; IR. $\nu_{\max }$ (KBr): 3321, 2955, 2925, 2853, 1693, 1669, 1598, 1540, 1278, $1169,835 \mathrm{~cm}^{-1}$; Elemental Analysis for $\mathrm{C}_{17} \mathrm{H}_{16} \mathrm{ClNO}_{4}$ Calcd. C, 61.18; H, 4.83; N, 4.20; Found C, 61.13; H, 4.77; N, 4.13.

(E)-Methyl (5-oxo-1,5-diphenylpent-1-en-3-yl)carbamate (4q, Table 2). Brownish viscous liquid; ${ }^{1} \mathrm{H}$ NMR $(400 \mathrm{MHz}$, $\left.\mathrm{CDCl}_{3}\right): \delta 3.34\left(2 \mathrm{H}, \mathrm{dd}, J=5.6,17.2 \mathrm{~Hz}, \mathrm{CH}_{2}\right), 3.67(3 \mathrm{H}$, s, $\left.\mathrm{COOCH}_{3}\right), 4.85-4.88(1 \mathrm{H}, \mathrm{m}, \mathrm{CH}), 5.69(1 \mathrm{H}, \mathrm{s}, \mathrm{NH})$, $6.33(1 \mathrm{H}, \mathrm{dd}, J=6.4,16 \mathrm{~Hz}, \mathrm{CH}=), 6.57(1 \mathrm{H}, \mathrm{d}, J=16 \mathrm{~Hz}$, $\mathrm{CH}=), 7.19-7.41(5 \mathrm{H}, \mathrm{m}, \operatorname{Ar}-\mathrm{H}), 7.46(1 \mathrm{H}, \mathrm{t}, J=7.6 \mathrm{~Hz}$, Ar-H), $7.56(2 \mathrm{H}, \mathrm{d}, J=7.6 \mathrm{~Hz}, \operatorname{Ar}-\mathrm{H}), 7.95(2 \mathrm{H}, \mathrm{d}, J=$ $7.2 \mathrm{~Hz}, \mathrm{Ph}$ ) ppm; ${ }^{13} \mathrm{C} \mathrm{NMR}\left(100 \mathrm{MHz}, \mathrm{CDCl}_{3}\right): \delta 43.1,50.0$, 52.2, 126.5, 127.7, 128.1, 128.5, 128.7, 131.1, 133.5, 136.4, 136.7, 156.4, 195.6 ppm; IR. $\nu_{\max }(\mathrm{KBr}): 3434,3023,2977$, $2895,1712,1686,1519,1424,1221,1045 \mathrm{~cm}^{-1}$; MS $\left(\mathrm{ES}^{+}\right)$for $\mathrm{C}_{19} \mathrm{H}_{19} \mathrm{NO}_{3} \mathrm{~m} / \mathrm{z}(\mathrm{M}+\mathrm{H})^{+} 310.14$, found $(\mathrm{M}+\mathrm{H})^{+} 310.5$; Elemental Analysis for $\mathrm{C}_{19} \mathrm{H}_{19} \mathrm{NO}_{3}$ Calcd. C, 73.77; H, 6.19; N, 4.53; Found C, 73.71; H, 6.21; N, 4.57.

Methyl 2-((3,4-dimethoxyphenyl) ((methoxycarbonyl)amino) methyl)-3-oxobutanoate (4r, Table 2). White solid; mp 140$144^{\circ} \mathrm{C} ;{ }^{1} \mathrm{H}$ NMR $\left(400 \mathrm{MHz},\left(\mathrm{CD}_{3}\right){ }_{2} \mathrm{CO}-\mathrm{d}_{6}\right): \delta 2.11(3 \mathrm{H}, \mathrm{s}$, $\left.\mathrm{COCH}_{3}\right), 3.40\left(3 \mathrm{H}, \mathrm{s}, \mathrm{COOCH}_{3}\right), 3.65\left(3 \mathrm{H}, \mathrm{s}, \mathrm{COOCH}_{3}\right)$, $3.67\left(6 \mathrm{H}, \mathrm{s}, \mathrm{COCH}_{3}\right), 4.11-4.12(1 \mathrm{H}, \mathrm{m}, \mathrm{CH}), 5.21-5.26$ $(1 \mathrm{H}, \mathrm{m}, \mathrm{CH}), 6.7-6.94(3 \mathrm{H}, \mathrm{m}, \mathrm{Ar}-\mathrm{H}) \mathrm{ppm} ;{ }^{13} \mathrm{C} \mathrm{NMR}$ $\left(100 \mathrm{MHz},\left(\mathrm{CD}_{3}\right){ }_{2} \mathrm{CO}-\mathrm{d}_{6}\right): \delta 30.2,49.8,52.3,52.4,52.5,63.3$, $108.9,110.4,111.1,118.6,126.9,130.1,132.0,156.3,167.7$, 
190.9 ppm; IR. $v_{\max }(\mathrm{KBr}): 3366,3050,3002,2960,2839$, $1714,1594,1519,1441,1026 \mathrm{~cm}^{-1}$; Elemental Analysis for $\mathrm{C}_{16} \mathrm{H}_{21} \mathrm{NO}_{7}$ Calcd. C, 56.63; H, 6.24; N, 4.13; Found C, 56.53; $\mathrm{H}, 6.28 ; \mathrm{N}, 4.18$.

Methyl2-(((methoxycarbonyl)amino) (3,4,5-trimethoxyphenyl) methyl)-3-oxobutanoate (4s, Table 2). White solid; mp 157$160^{\circ} \mathrm{C} ;{ }^{1} \mathrm{H} \mathrm{NMR}\left(400 \mathrm{MHz}, \mathrm{CDCl}_{3}\right): \delta 2.18\left(3 \mathrm{H}, \mathrm{s}, \mathrm{CH}_{3}\right)$, $3.67\left(3 \mathrm{H}, \mathrm{s}, \mathrm{COOCH}_{3}\right), 3.72\left(3 \mathrm{H}, \mathrm{s}, \mathrm{COOCH}_{3}\right), 3.81(3 \mathrm{H}$, $\left.\mathrm{s}, \mathrm{COCH}_{3}\right), 3.85\left(6 \mathrm{H}, \mathrm{s}, \mathrm{COCH}_{3}\right), 4.01(1 \mathrm{H}, \mathrm{d}, J=5.2 \mathrm{~Hz}$, $\mathrm{CH}), 5.37-5.40(1 \mathrm{H}, \mathrm{m}, \mathrm{CH}), 6.16(1 \mathrm{H}, \mathrm{d}, J=8.8 \mathrm{~Hz}, \mathrm{NH})$, $6.50(2 \mathrm{H}, \mathrm{s}, \mathrm{Ar}-\mathrm{H}) \mathrm{ppm} ;{ }^{13} \mathrm{C} \mathrm{NMR}\left(100 \mathrm{MHz}, \mathrm{CDCl}_{3}\right): \delta$ $31.0,52.4,52.9,54.7,56.3,60.8,62.9,103.6,106.7,135.2$, 137.5, 153.3, 167.6, 203.4 ppm; IR. $v_{\max }(\mathrm{KBr}): 3370,2995$, 2956, 2927, 2851, 1728, 1711, 1592, 1540, 1297, $1127 \mathrm{~cm}^{-1}$; MS $\left(\mathrm{ES}^{+}\right)$for $\mathrm{C}_{17} \mathrm{H}_{23} \mathrm{NO}_{8} \mathrm{~m} / \mathrm{z}(\mathrm{M}+\mathrm{Na})^{+} 392.14$, found $(\mathrm{M}$ $+\mathrm{Na})^{+}$392.4; Elemental Analysis for $\mathrm{C}_{17} \mathrm{H}_{23} \mathrm{NO}_{8}$ Calcd. C, 55.28; H, 6.28; N, 3.79; Found C, 55.31; H, 6.24; N, 3.83.

Diethyl 2-((3-chlorophenylamino)(phenyl)methyl)malonate (4t, Table 2). White solid; mp $113-114^{\circ} \mathrm{C} ;{ }^{1} \mathrm{H}$ NMR $(400 \mathrm{MHz}$, $\left.\mathrm{CDCl}_{3}\right): \delta 1.09\left(3 \mathrm{H}, \mathrm{t}, J=7.2 \mathrm{~Hz}, \mathrm{CH}_{3}\right), 1.16(3 \mathrm{H}, \mathrm{t}, J=$ $\left.7.2 \mathrm{~Hz}, \mathrm{CH}_{3}\right), 3.86(1 \mathrm{H}, \mathrm{d}, J=5.2 \mathrm{~Hz}, \mathrm{CH}), 4.12(2 \mathrm{H}, \mathrm{q}, J=$ $\left.7.2 \mathrm{~Hz}, \mathrm{CH}_{2}\right), 4.04\left(2 \mathrm{H}, \mathrm{q}, J=7.2 \mathrm{~Hz}, \mathrm{CH}_{2}\right), 5.22(1 \mathrm{H}, \mathrm{d}, J=$ $5.6 \mathrm{~Hz}, \mathrm{CH}), 5.64$ (1H, br. s, NH), $6.49(1 \mathrm{H}, \mathrm{d}, J=8.8 \mathrm{~Hz}, \mathrm{Ar}-$ $\mathrm{H}), 6.59(1 \mathrm{H}, \mathrm{s}, \mathrm{Ar}-\mathrm{H}), 6.70(1 \mathrm{H}, \mathrm{d}, J=8.8 \mathrm{~Hz}, \mathrm{Ar}-\mathrm{H}), 7.00$ $(1 \mathrm{H}, \mathrm{t}, J=8.8 \mathrm{~Hz}, \mathrm{Ar}-\mathrm{H}), 7.26-7.35(5 \mathrm{H}, \mathrm{m}, \mathrm{Ar}-\mathrm{H}) \mathrm{ppm} ;{ }^{13} \mathrm{C}$ NMR $\left(100 \mathrm{MHz}, \mathrm{CDCl}_{3}\right): \delta 13.8,14.0,56.7,57.8,61.5,61.8$, $111.8,113.3,117.6,126.5,127.8,128.6,130.0,134.9,139.0$, 147.8, 167.0, 168.0 ppm; IR. $\nu_{\max }(\mathrm{KBr}): 3376,2990,2980$, 2933, 2800, 1753, 1568, 1540,1309, $1196 \mathrm{~cm}^{-1}$; Elemental analysis for $\mathrm{C}_{20} \mathrm{H}_{22} \mathrm{ClNO}_{4}$ Calcd. C 63.91; $\mathrm{H}$ 5.90; $\mathrm{N}$ 3.73; Found C 63.71; H 5.83; N 3.61.

\section{Acknowledgments}

M. Rajbangshi thanks the UGC-RGNF for financial assistance and SAIF and NEHU for providing analytical supports.

\section{References}

[1] J. Barluenga, A. L. Viado, E. Aguilar, S. Fustero, and B. Olano, "1,3-amino alcohols from 4-amino-1-aza dienes. Diastereoand enantioselective approach to the four diastereoisomers of the N-terminal amino acid component of nikkomycins B and BX," Journal of Organic Chemistry, vol. 58, no. 22, pp. 59725975, 1993.

[2] D. Enders, M. Moser, G. Geibel, and M. C. Laufer, "Diastereoand enantioselective synthesis of differently N,O-protected 1,3-amino alcohols with three neighbouring stereogenic centers," Synthesis, no. 12, pp. 2040-2046, 2004.

[3] M. Mukhopadhyay, B. Bhatia, and J. Iqbal, "Cobalt catalyzed multiple component condensation route to $\beta$-acetamido carbonyl compound libraries," Tetrahedron Letters, vol. 38, no. 6, pp. 1083-1086, 1997.

[4] K. Kobinata, M. Uramoto, M. Nishii, H. Kusakabe, G. Nakamura, and K. Isono, "Neopolyoxins A, B, and C, new chitin synthetase inhibitors," Agricultural and Biological Chemistry, vol. 44 , p. $1709,1980$.
[5] U. Daehn, H. Hagenmaier, H. Hoehne, W. A. Koenig, G. Wolf, and H. Zaehner, "Metabolic products of microorganisms. 154. Nikkomycin, a new inhibitor of fungal chitin synthesis," Archives of Microbiology, vol. 107, no. 2, pp. 143-160, 1976.

[6] H. R. Shaterian, A. Hosseinian, and M. Ghashang, "Silicasupported perchloric acid $\left(\mathrm{HClO}_{3}-\mathrm{SiO}_{2}\right)$ : an efficient catalyst for the preparation of $\beta$-amido carbonyl compounds using multicomponent reactions," Synthetic Communications, vol. 38, no. 21, pp. 3766-3777, 2008.

[7] M. M. Khodaei, A. R. Khosropour, and P. Fattahpour, "A modified procedure for the Dakin-West reaction: an efficient and convenient method for a one-pot synthesis of $\beta$-acetamido ketones using silica sulfuric acid as catalyst," Tetrahedron Letters, vol. 46, no. 12, pp. 2105-2108, 2005.

[8] A. T. Khan, T. Parvin, and L. H. Choudhury, "Effects of substituents in the $\beta$-position of 1,3-dicarbonyl compounds in bromodimethylsulfonium bromide-catalyzed multicomponent reactions: a facile access to functionalized piperidines," Journal of Organic Chemistry, vol. 73, no. 21, pp. 8398-8402, 2008.

[9] M. M. Heravi, L. Ranjbar, F. Derikvand, and F. F. Bamoharram, "A modified and green Dakin-West reaction: an efficient and convenient method for a one-pot synthesis of $\beta$-acetamido carbonyl compounds," Journal of Molecular Catalysis A, vol. 271, no. 1-2, pp. 28-31, 2007.

[10] B. Das, K. R. Reddy, Y. Srinivas, and R. A. Kumar, "One-pot multicomponent synthesis of $\beta$-acetamidoketones catalysed by pTSA," Canadian Journal of Chemistry, vol. 85, no. 7-8, pp. 479-482, 2007.

[11] X. Wang, H. Mao, Y. Yu, X. Zhu, and C. Zhu, "Samarium triiodide-catalyzed formation of Mannich-type products by amidoalkylation of 1,3-dicarbonyl compounds," Synthetic Communications, vol. 37, no. 21, pp. 3751-3758, 2007.

[12] B. Das and K. R. Reddy, "Facile one-pot multicomponent synthesis of $\beta$-acetamido ketones with Amberlyst-15 as heterogeneous catalyst," Helvetica Chimica Acta, vol. 89, no. 12, pp. 3109-3111, 2006.

[13] L. W. Xu, C. G. Xia, and L. Li, "Transition metal salt-catalyzed direct three-component Mannich reactions of aldehydes, ketones, and carbamates: efficient synthesis of $\mathrm{N}$-protected $\beta$ aryl- $\beta$-amino ketone compounds," Journal of Organic Chemistry, vol. 69, no. 24, pp. 8482-8484, 2004.

[14] B. A. Bunin, The Combinatorial Index, Academic Press, New York, NY, USA, 1998.

[15] A. W. Czarnik and S. H. DeWitt, Eds., A Practical Guide to Combinatorial Chemistry, American Chemical Society, Washington, DC, USA, 1997.

[16] K. C. Nicolaou, R. Hanko, and W. Hartwig, Handbook of Combinatorial Chemistry, Wiley-VCH, Weinheim, Germany, 2002.

[17] I. Saxena, D. C. Borah, and J. C. Sarma, "Three component condensations catalyzed by iodine-alumina for the synthesis of substituted 3,4-dihydropyrimidin-2(1H)-ones under microwave irradiation and solvent-free conditions," Tetrahedron Letters, vol. 46, no. 7, pp. 1159-1160, 2005.

[18] S. Caddick, "Microwave assisted organic reactions," Tetrahedron, vol. 51, no. 38, pp. 10403-10432, 1995.

[19] D. M. P. Mingos, "Microwaves in chemical synthesis," Chemistry and Industry, vol. 596, 1994.

[20] M. Larhed, C. Moberg, and A. Hallberg, "Microwaveaccelerated homogeneous catalysis in organic chemistry," Accounts of Chemical Research, vol. 35, no. 9, pp. 717-727, 2002. 
[21] P. Phukan, D. Kataki, and P. Chakraborty, "Direct synthesis of Cbz-protected $\beta$-amino ketones by iodine-catalyzed threecomponent condensation of aldehydes, ketones and benzyl carbamate," Tetrahedron Letters, vol. 47, no. 31, pp. 5523$5525,2006$.

[22] M. R. Rohman, M. Rajbangshi, B. M. Laloo, P. R. Sahu, and B. Myrboh, "Iodine-alumina as an efficient and useful catalyst for the regeneration of carbonyl functionality from the corresponding 1,3-oxathiolanes and 1,3-dithiolanes in aqueous system," Tetrahedron Letters, vol. 51, no. 21, pp. 28622864, 2010.

[23] Z. Li, X. Ma, J. Liu, X. Feng, G. Tian, and A. Zhu, "Silicasupported aluminum chloride: a recyclable and reusable catalyst for one-pot three-component Mannich-type reactions," Journal of Molecular Catalysis A, vol. 272, no. 1-2, pp. 132-135, 2007.

[24] N. Deka and J. C. Sarma, "Highly efficient dithioacetalization of carbonyl compounds catalyzed with iodine supported on neutral alumina," Chemistry Letters, no. 8, pp. 794-795, 2001. 


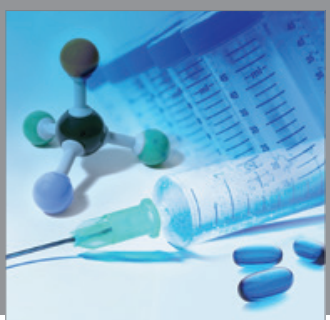

International Journal of

Medicinal Chemistry

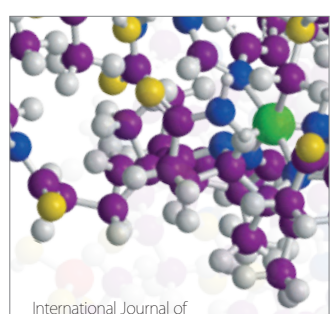

Carbohydrate Chemistry

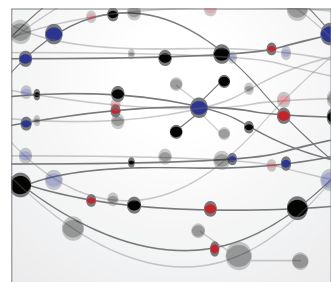

The Scientific World Journal
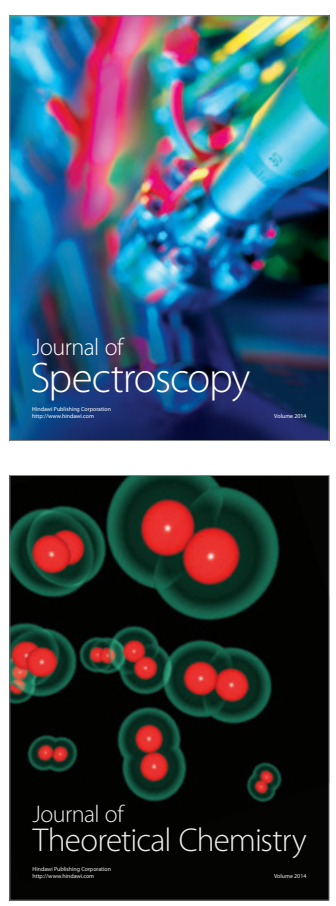
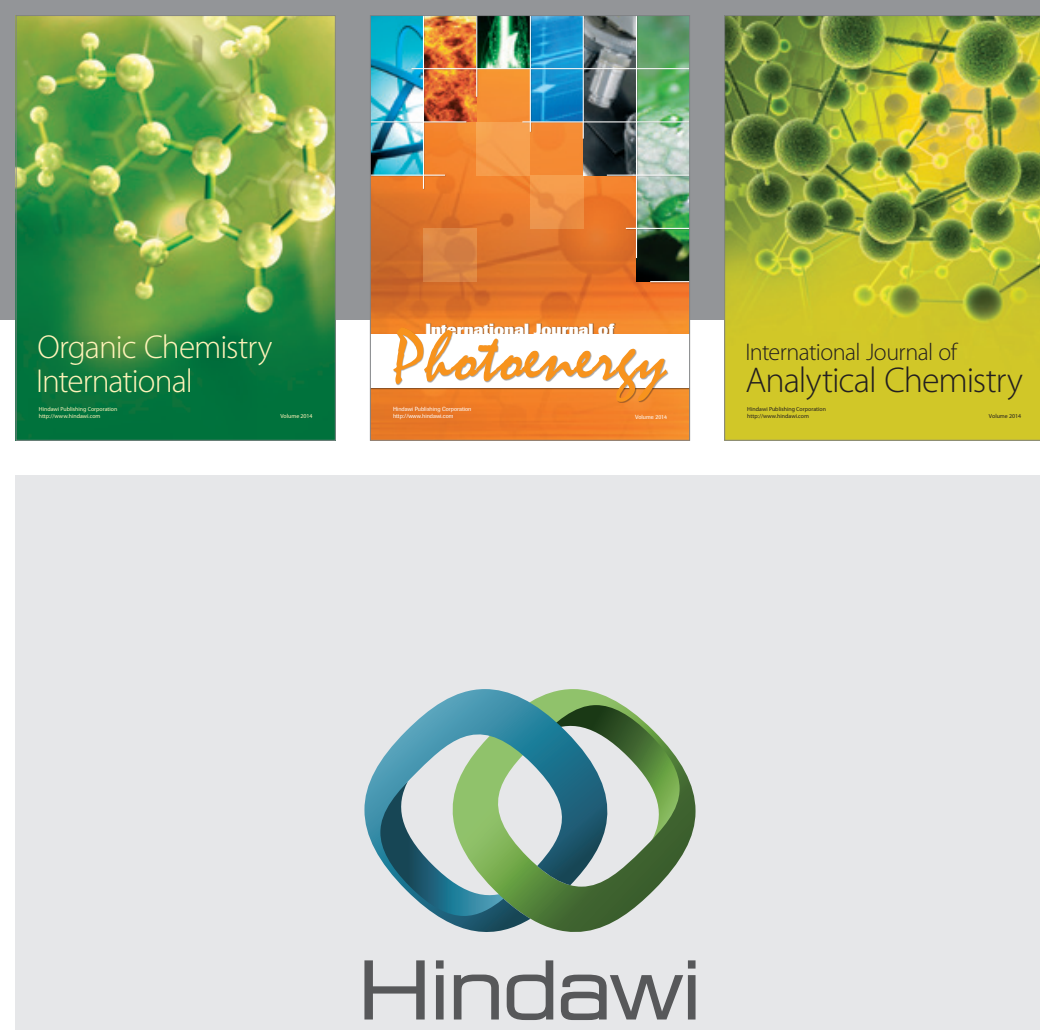

Submit your manuscripts at

http://www.hindawi.com
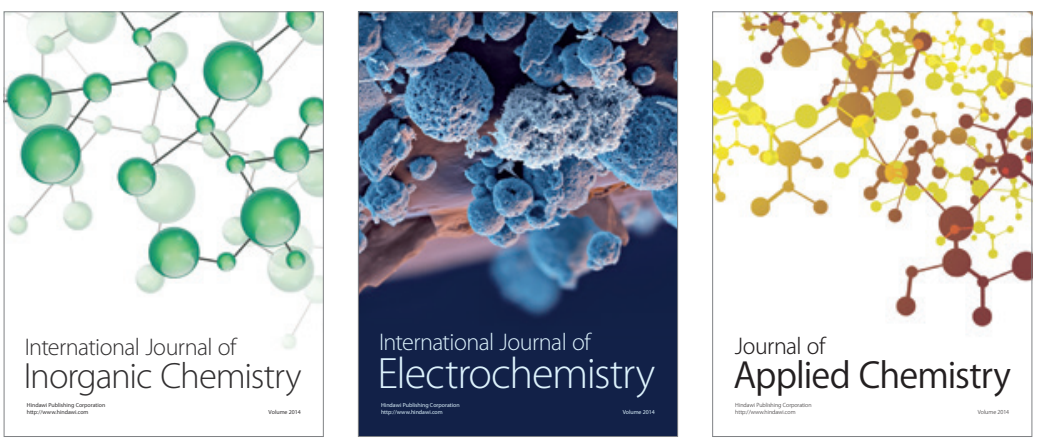

Journal of

Applied Chemistry
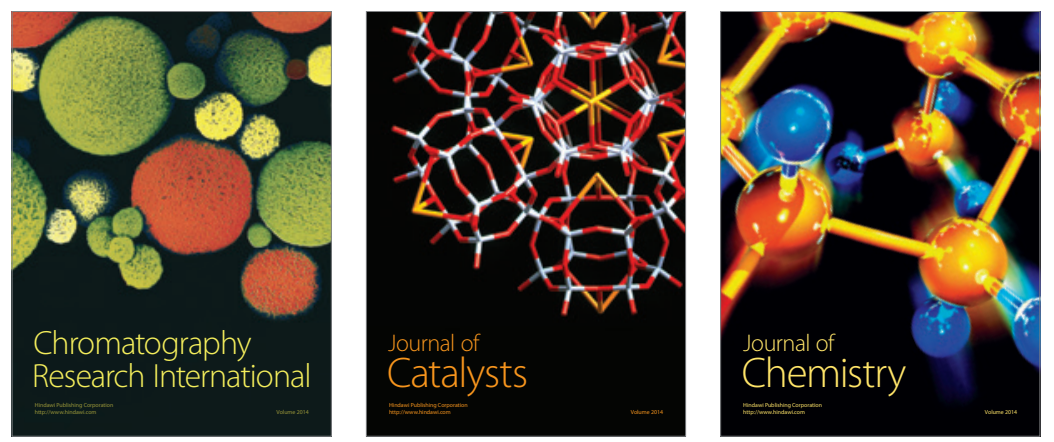
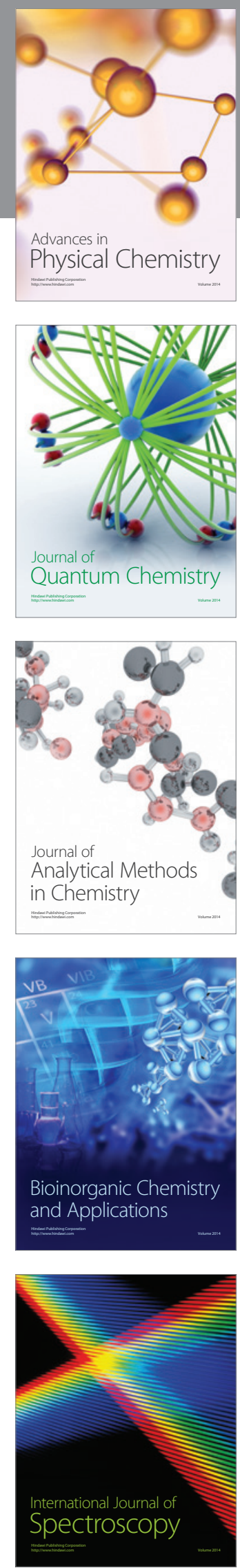